\title{
Raman spectral signatures of human liver perfusates correlate with oxidation reduction potential
}

\author{
RAPHAEL BAR-OR ${ }^{1,3}$, LEONARD T. RAEL ${ }^{1,3}$, C. GERALD CURTIS ${ }^{4}$, CHARLES W. MAINS $^{5}$, \\ DENETTA S. SLONE ${ }^{1}$ and DAVID BAR-OR ${ }^{1,2,3,5}$ \\ ${ }^{1}$ Trauma Research Laboratory, and ${ }^{2}$ Emergency Department, Swedish Medical Center, Englewood, CO 80113; \\ ${ }^{3}$ DMI BioSciences Inc., Greenwood Village, CO 80111; ${ }^{4}$ Bowman Research, Des Plaines, IL 60018; \\ ${ }^{5}$ Trauma Research, St. Anthony Central Hospital, Denver, CO 80204, USA
}

Received October 27, 2008; Accepted December 17, 2008

DOI: $10.3892 / \mathrm{mmr} 00000080$

\begin{abstract}
Biomarkers for the early detection of liver toxicity are crucial in drug development for assessing the safety of a new drug. Oxidation reduction potential (ORP) is an overall measure of the oxidative stress to which a biological component is subjected and correlates with organ dysfunction. Raman spectroscopy is a non-invasive method that we employed to analyze the perfusates of five normothermic human livers perfused with the known toxin $\alpha$-naphthylisothiocyanate. Spectral signatures were generated using principle component analysis (PCA) coupled with stepwise linear regression of the first several PCA coefficients to the ORP. The Raman signatures correlated to the measured ORP with an $\mathrm{r}^{2}$ of 0.854 . This study demonstrated the utility of this technique in determining the presence of liver toxicity as reflected by increasing ORP. Real-time, non-invasive monitoring of normothermic perfusates of human livers using processed Raman spectra has the potential to predict drug toxicity and organ viability for transplantation.
\end{abstract}

\section{Introduction}

Species and gender differences in the fate and effects of drugs and their metabolites are well documented. In many instances, these differences have been attributed to variations in the uptake, accumulation or concentration of enzymes and cofactors, particularly in the liver. Currently, attempts to predict the fate and effects of drug candidates in the human liver, prior to human clinical trials, utilize in vitro data from tissue slices, isolated cells, homogenates, S9 fraction or subcellular particles (i.e., microsomes, mitochondria). Although these methods have a very high throughput, the drug-related data does not always

Correspondence to: Dr David Bar-Or, Trauma Research Laboratory, Swedish Medical Center, 501 E. Hampden Avenue, Rm. 4-454, Englewood, CO 80113, USA

E-mail: dbaror@dmibio.com

Key words: Raman spectroscopy, oxidation reduction potential, organ perfusion accurately reflect occurrences in the intact human liver. The use of isolated perfused human livers (rejected for transplant but ethically donated for research) is a technology that overcomes many of the shortcomings inherent to in vitro systems. Perfused livers maintained under near normal physiological and biochemical conditions used for drug safety trials are likely to result in improved prediction of liver toxicity in vivo.

In this study, we used a hepatotoxin, $\alpha$-naphthylisothiocyanate (ANIT), which induces the separation of intercellular tight junctions that seal the bile canaliculi (1). ANIT causes the impairment of biliary secretion, resulting in the retention of bile acids (which are the major route of cholesterol elimination) and bilirubin (which is therefore prevented from forming glucuronides and being eliminated). The resulting damage is referred to as cholangiodestructive cholestasis, and is associated with elevations in serum aspartate aminotransferase and $\gamma$-glutamyltransferase activities (2). The canalicular lesions induce neutrophil infiltration and release cytotoxic proteases and reactive oxygen species, thereby damaging the hepatocytes further and setting up a toxic chain reaction (3). Raman spectroscopy was used to detect spectral signatures in perfusates of a human liver perfusion model. Similar to infrared (IR) spectroscopy, Raman spectroscopy measures the vibrational modes in a system and provides complementary data to IR. For the purposes of correlation, oxidation reduction potential (ORP) was measured in the perfusates using a previously described method (4).

\section{Materials and methods}

Organ perfusion. Five human livers (Table I), rejected for transplantation but ethically donated for research, were removed from cold storage and flushed with a proprietary buffer via the hepatic artery $(1000 \mathrm{ml})$ and portal vein (1000 ml).

The livers were attached to the perfusion apparatus and perfused via both vessels with oxygenated perfusates, comprised of matched human erythrocytes suspended in buffer containing $6 \% \mathrm{w} / \mathrm{v}$ human albumin (Grifols Biologicals Inc., Los Angeles, CA, USA). Once the core temperature reached $35^{\circ} \mathrm{C}$ and perfusion flows were stable (usually within $1-2 \mathrm{~h}$ ), the perfusates were replaced with 2 liters of fresh perfusates. After a short re-equilibration time (15-20 min), ANIT was 
Table I. Donor demographics.

\begin{tabular}{lccccc}
\hline Perfusion no. & Control & 1 & 2 & 3 & 4 \\
\hline Gender & Female & Male & Male & Male & Male \\
Age (years) & 54 & 50 & 31 & 20 & 22 \\
Body weight (kg) & 109 & 82.6 & 128 & 91 & 90 \\
Blood group & $\mathrm{O}$ & $\mathrm{A}^{+}$ & $\mathrm{A}^{-}$ & $\mathrm{A}^{+}$ & $\mathrm{A}^{+}$ \\
Cold ischemia time (h) & 15.5 & 9 & 15 & 14 & 12 \\
\hline
\end{tabular}

slowly added to the perfusates as follows: $\mathrm{t}=0,2 \mathrm{mg}$; $\mathrm{t}=30 \mathrm{~min}$, $4 \mathrm{mg}$; $\mathrm{t}=60 \mathrm{~min}, 6 \mathrm{mg}$; $\mathrm{t}=90 \mathrm{~min}, 9 \mathrm{mg}$; and $\mathrm{t}=120 \mathrm{~min}, 20 \mathrm{mg}$. Bile salts were added at 30 min post-dosing with ANIT and every 30 min thereafter. In the control liver, the dosing with ANIT was omitted. A total of 47 samples was collected for the experiments.

Perfusate aliquots $(10 \mathrm{ml})$ were taken every $30 \mathrm{~min}$ starting at $\mathrm{t}=0$ and analyzed for blood chemistry/biochemistry. A portion of each aliquot was centrifuged and the supernatant (plasma) removed and flash frozen for analysis by Raman spectroscopy and ORP.

Oxidation reduction potential. ORP measurements were recorded using an MI-800/410 cm Redox Electrode (Microelectrodes Inc., Bedford, NH, USA) connected to an HI4222 pH/mV/temperature bench meter (Hanna Instruments, Woonsocket, RI, USA). Perfusion samples were thawed at room temperature, and the ORP electrode was immersed in the perfusate. A reading was recorded in millivolts $(\mathrm{mV})$ after the ORP value was stable for $5 \mathrm{sec}$.

Raman spectroscopy. Raman spectra for each sample were obtained by placing an unprocessed thawed perfusate sample in a quartz cuvette and measuring a Raman shift spectrum versus a water control using a Raman Systems R3000QE spectrometer equipped with a 785-nm laser with a maximum power output of $300 \mathrm{~mW}$ (Raman Systems Inc., Austin, TX). A custom needle immersion probe was used in addition to a filtered fixed focus probe. Raman shifts from 200 to $1800 \mathrm{~cm}^{-1}$ were acquired. The raw spectra were imported to Matlab R2006b (Mathworks, Natick, MA, USA) for analysis. Each of the spectra was imported as a vector $s_{\mathrm{i}}$, with all of the spectra together in the matrix $S$ as columns.
Table III. Net change in perfusate biochemistry per hour of perfusion.

\begin{tabular}{lcccc}
\hline $\begin{array}{l}\text { Perfusion } \\
\text { no. }\end{array}$ & $\begin{array}{c}\text { Glucose } \\
(\text { mmoles/h) }\end{array}$ & $\begin{array}{c}\text { Lactate } \\
(\text { mmoles/h })\end{array}$ & $\begin{array}{c}\mathrm{K}^{+} \\
(\text {mmoles/h })\end{array}$ & $\begin{array}{c}\text { ALT } \\
(\mathrm{U} / \mathrm{l} / \mathrm{h})\end{array}$ \\
\hline Control & 7.50 & 1.9 & 1.80 & 490 \\
Test 1 & 6.70 & 5.3 & 5.20 & 962 \\
Test 2 & 0.83 & 5.1 & 9.00 & $»$ \\
Test 3 & -1.75 & 0.9 & 2.38 & 244 \\
Test 4 & 0.81 & 1.6 & 2.56 & $»$ \\
\hline
\end{tabular}

», too high to measure.

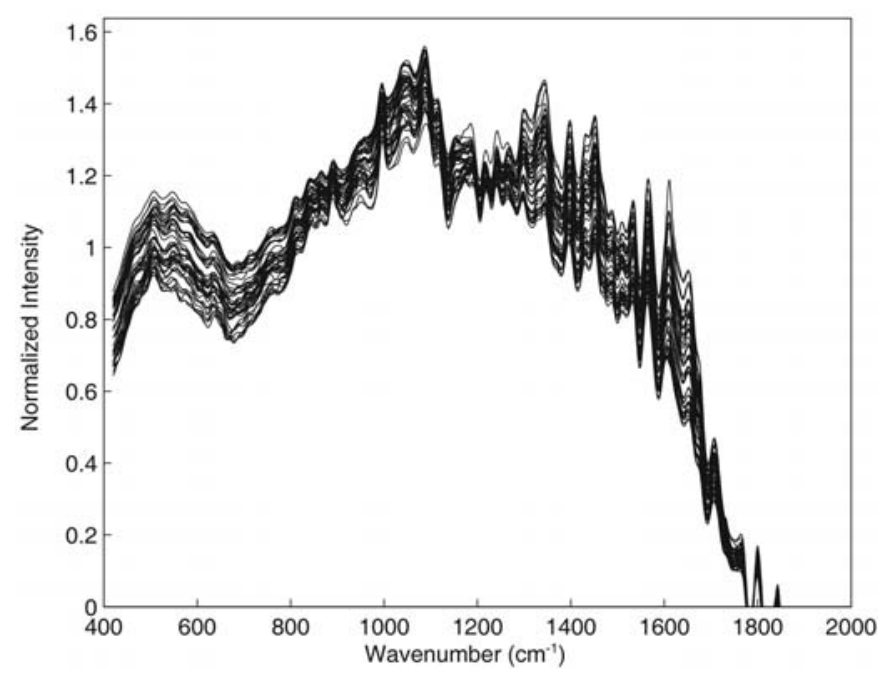

Figure 1. Normalized Raman spectra for all samples.

The system resolution was approximately $6 \mathrm{~cm}^{-1}$ FWHM (full width half maximum). It was therefore reasonable to assume that any narrower features were noise and could be filtered. The spectra were smoothed using a 2-pass SavitskyGolay filter with a 4 channel window $\left(\sim 5.3 \mathrm{~cm}^{-1}\right)$ and a third order polynomial. This level and type of smoothing removes high frequency noise while preserving local spectral features (5). Background drift and broadband fluorescence were removed using a weighted least squares fifth order polynomial

Table II. Physiological measurements.

\begin{tabular}{lccccc}
\hline Parameter & Control & Perfusion 1 & Perfusion 2 & Perfusion 3 & Perfusion 4 \\
\hline Perfusion time post-dose (h) & 4 & 4 & 4 & 4 & 3 \\
Arterial pressure (mmHg) & $26 \pm 3.4$ & $24.7 \pm 8.5$ & $15.0 \pm 4.5$ & $18.6 \pm 2.8$ & $30.0 \pm 8.8$ \\
Venous pressure (mmHg) & $13 \pm 1.4$ & $19.3 \pm 4.3$ & $13.0 \pm 0.9$ & $7.8 \pm 4.7$ & $20.7 \pm 23.1$ \\
Arterial flow (ml/min/g) & $99 \pm 0.0$ & $266 \pm 73.0$ & $150 \pm 0.0$ & $200 \pm 0.0$ & $60 \pm 0.0$ \\
Venous flow (ml/min/g) & $600 \pm 0.0$ & $715 \pm 114.0$ & $600 \pm 0.0$ & $550 \pm 0.0$ & $585 \pm 181.0$ \\
Packed cell volume $(\%)$ & $15 \pm 0.7$ & $19.2 \pm 0.4$ & $15.7 \pm 1.1$ & $14.6 \pm 0.7$ & $15.3 \pm 0.8$ \\
Temperature $\left({ }^{\circ} \mathrm{C}\right)$ & $36.8 \pm 0.5$ & $36.6 \pm 0.7$ & $36.5 \pm 0.2$ & $36.8 \pm 0.2$ & $35.0 \pm 2.7$ \\
pH & $7.08 \pm 0.1$ & $7.20 \pm 0.2$ & $7.21 \pm 0.2$ & $7.53 \pm 0.2$ & $6.91 \pm 0.08$ \\
\hline
\end{tabular}



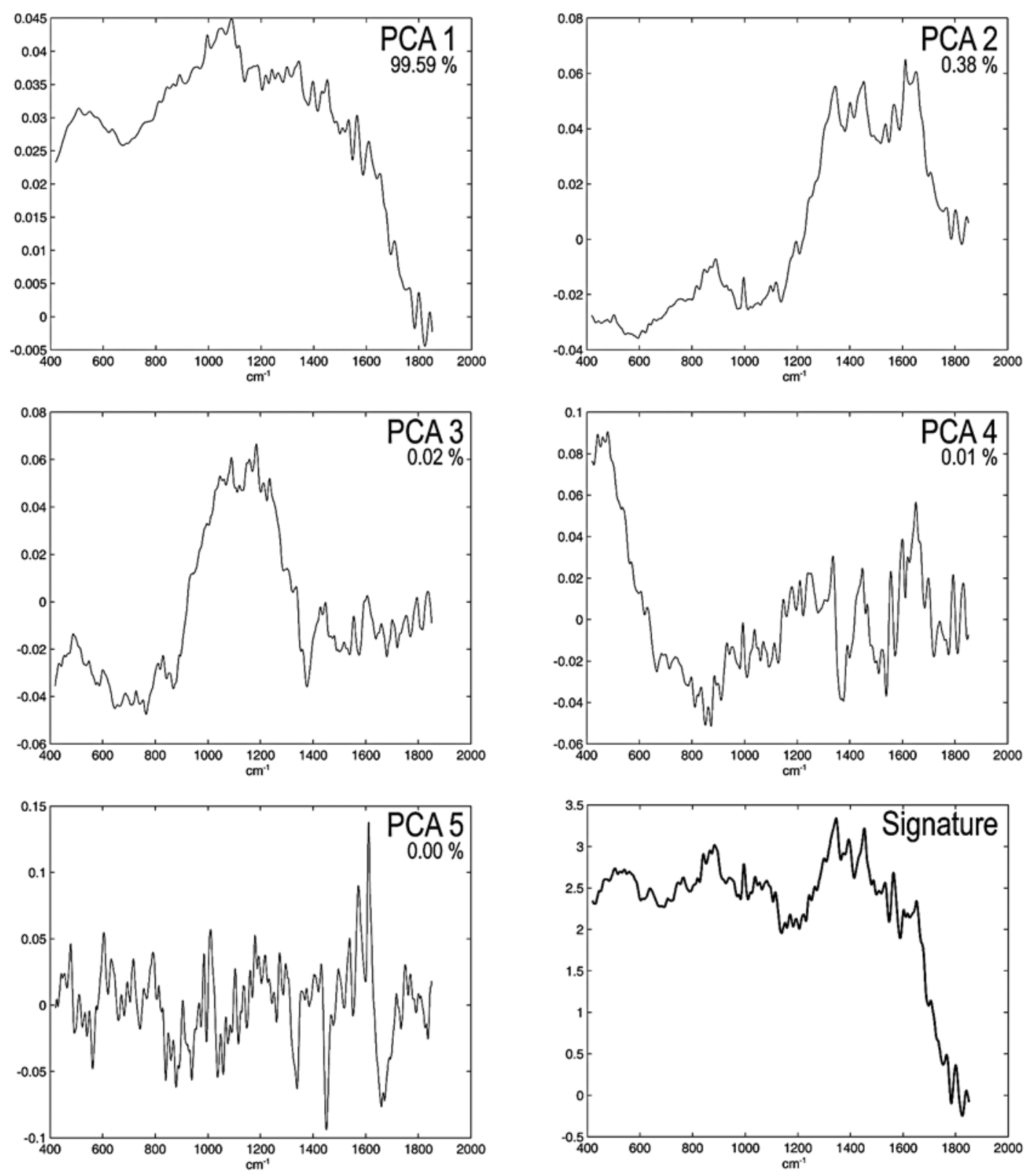

Figure 2. Principle component analysis (PCA) loadings for the first 5 principle components and the Raman regression model of ORP.

curve fit that weights heavily against negative residuals. All spectra were intensity normalized using the 1-norm:

$$
\left(s \dot{\mathrm{i}}=\frac{s_{\mathrm{i}}}{\left\|s_{\mathrm{i}}\right\|_{1}}\right)
$$

This removed any illumination and capture efficiency variations between samples.

Spectra were analyzed using principle component analysis (PCA) to reduce the dimensionality of each of the signals while maintaining the variability of the whole data set. Briefly, PCA is an orthogonal change of basis such that the components are ordered by the amount of variability that they explain between samples. Generally, due to the high degree of intervariable dependence in the data, it is possible to reproduce the signals accurately using only the first several components, the effect of which is a drastic reduction in the dimensionality of the data $(6,7)$. The signals were analyzed for possible signatures by looking at the coefficients of the first several principle components (those that represent the majority of the variability) and relating the coefficients to perfusion time and ORP. A linear stepwise regression was performed on the first several principle component coefficients against the ORP measurements to find a model that predicts ORP using the Raman data alone. A significance value of 0.05 was used as a maximal variable inclusion criterion, while 0.10 was used as a minimum variable removal criterion.

\section{Results}

Blood chemistry/biochemistry. Physiological parameters measured during the 4-h perfusion of the control liver and test livers dosed with ANIT are shown in Table II. Flow rates shown were expressed as means throughout the perfusion and were stable for some of the experiments $( \pm 0)$. Throughout the 

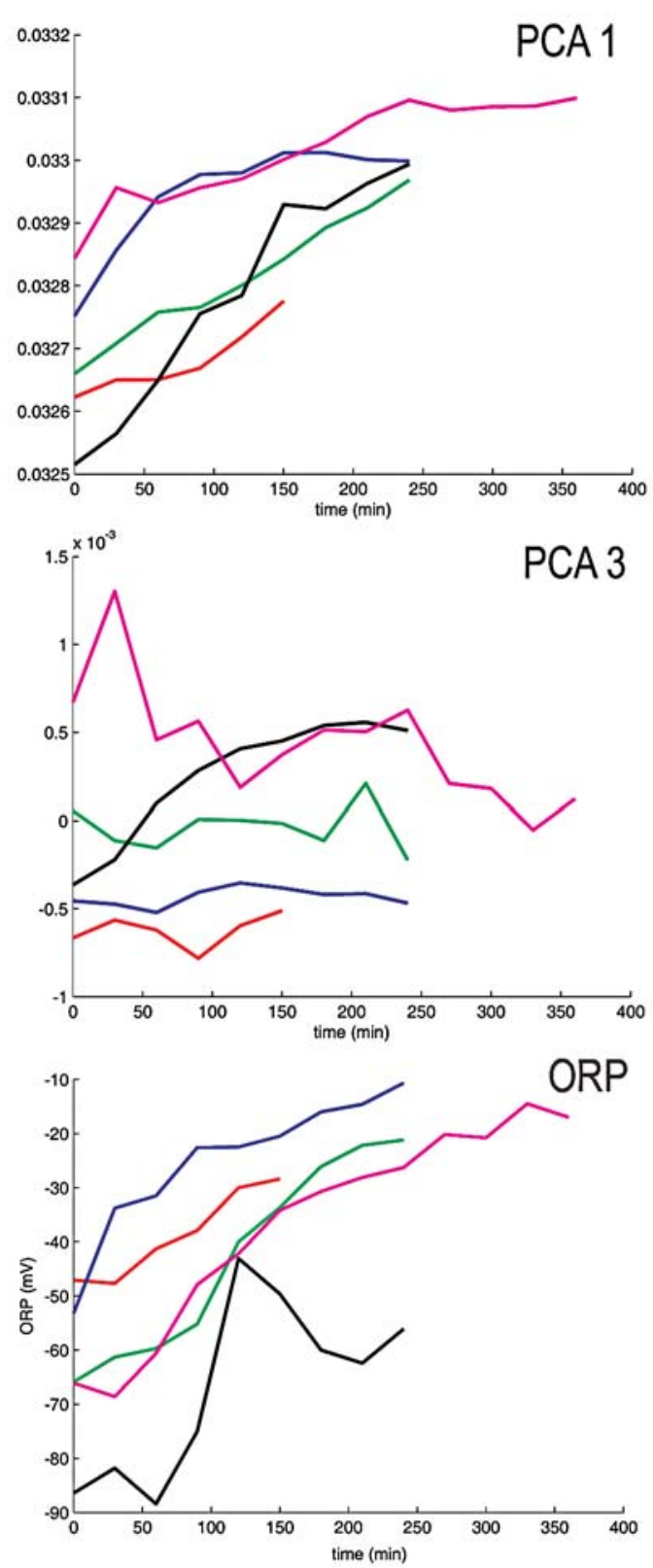
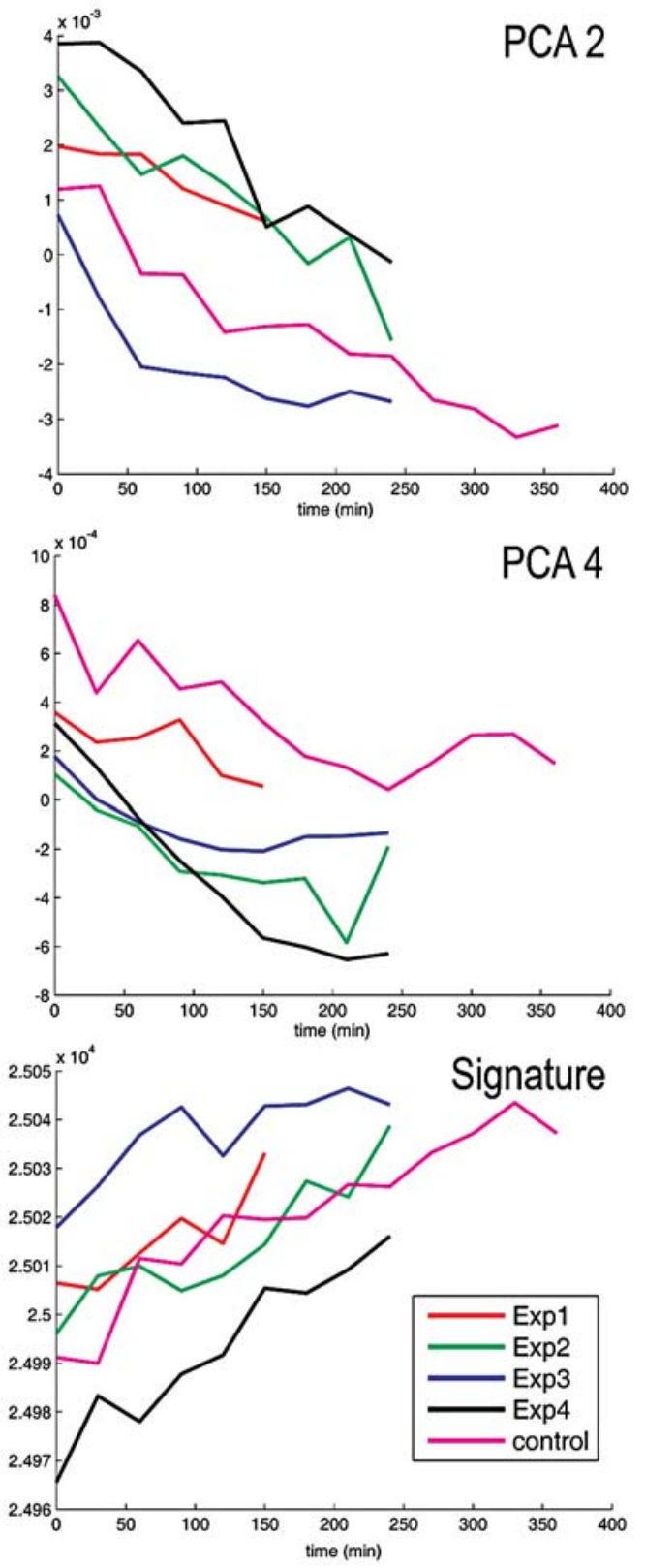

Figure 3. Temporal plots of the first 4 principle component analysis (PCA) coefficients, as well as oxidation reduction potential (ORP) and the Raman regression model of ORP.

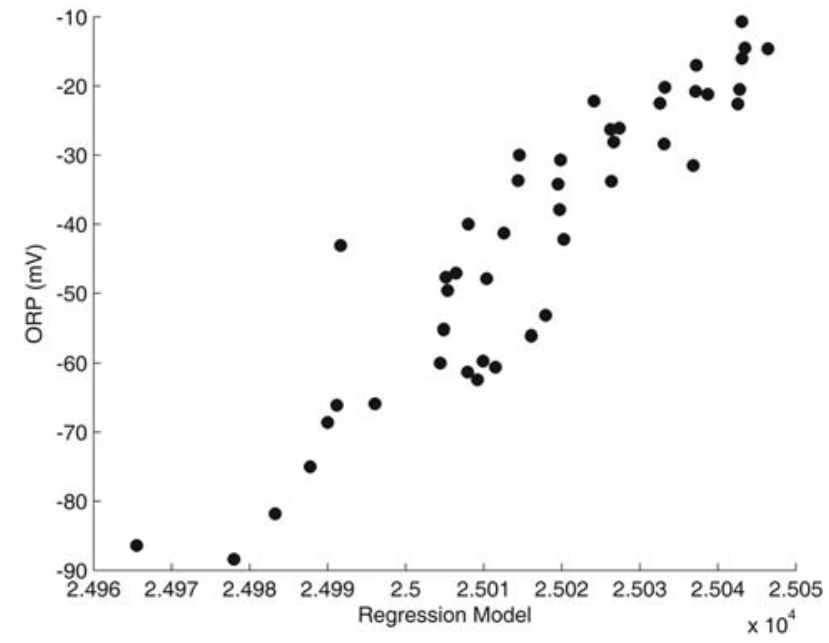

Figure 4. Correlation between oxidation reduction potential (ORP) and the Raman regression model. control and test perfusions, there was no significant change in the perfusates $\mathrm{Na}^{+}, \mathrm{Ca}^{2+}, \mathrm{Cl}^{-}$or osmolarity (data not shown). In contrast, there were time-dependent changes in the perfusates glucose, lactate, potassium and ALT (Table III).

Raman spectroscopy and oxidation reduction potential. The perfusates appeared to have distinct and reproducible Raman signals (Fig. 1). It was possible to capture $99.9 \%$ of the variation in these samples, with only 4 principle components suggesting that, while the data was captured in a thousand channels, the actual dimensionality of the variation space of these samples was $\sim 4$. The shapes, as well as the amount of variability captured for the first 5 principle components, are shown in Fig. 2. Note that the 5th PCA appears to be noise and explains $<10^{-4} \%$ of the total variation.

Plots of the temporal relationship of the PCA coefficients are shown in Fig. 3. The coefficients of PCA 1-4 vary smoothly with perfusion time. It appears that the control experiment 
stands out prominently in PCA 4, starting out much higher than the dosed experiments and converging to the level of the other experiments over time. This finding suggests that PCA 4 may be a good surrogate for 'health' if we assume that the non-dosed control liver is healthier than the dosed livers, and that by the end of the perfusion it is beginning to fail. PCA 1 shows an overall increase over time, with the control experiment and experiment 3 generally being higher than the others.

Oxidative stress is one of the primary reasons perfused organs fail $(8,9)$. All of the perfusate samples showed a marked increase in ORP with perfusion time (Fig. 3). This indicates a loss of reducing potential and probable ischemia during the course of perfusion. Stepwise regression of the ORP with the PCA coefficients accepted the first 4 principle components into the model and produced a strong linear correlation $\left(r^{2}=0.854\right)$ (Fig. 4) with an associated model p-value of $<10^{-14}$

\section{Discussion}

In drug development, the safety of compounds with lead status is evaluated using available in vitro and animal models. Following in vitro testing, compounds are evaluated in a rising dose study, usually of 7-14 days, to establish the dose at which signs of toxicity are observed. Initial in vivo toxicity testing is carried out for 14-90 days; the former is intended to provide sufficient data for a single Phase I clinical study, and the latter for a full clinical evaluation. Such investigations are time consuming (9 months for a 14-day study and 2-3 years to complete a 90-day study), requiring kilogram quantities of a drug substance. Despite these studies, the unexpected occurrence of idiosyncratic drug reactions during late clinical trials or after the drug has been released can lead to severe restrictions in use or even withdrawal. Liver toxicity is the most common reason drugs fail and are withdrawn by regulatory authorities. This was the rationale for initiating a research program to develop whole human livers (ethically donated for transplantation and research but rejected for transplantation) to determine whether drug-induced hepatic toxicity can be predicted in real-time after dosing into the blood.

Thus far, ex-vivo organ perfusion experiments have relied on physical parameter measurements and offline chemical assays to assess the viability of each organ throughout the normothermic perfusion period $(10,11)$. These include the release of a host of potential biomarkers, including liver enzymes (AST, ALT, LDH and GGT) into the perfusate. However, these enzymes can be released from the tissue by simple manual manipulation $(12,13)$. Consequently, their utility in evaluating the hepatotoxicity of drugs and drug candidates ex vivo is questionable. Clearly, the ability to assess the real-time status of each perfused human liver before and after dosing with drug candidates using an online, non-invasive technique would be beneficial.

Biomarkers for the early detection of toxicity hold the promise of improving failure rates in drug development. Three markers found to have exceptional predictive performance towards liver toxicity and/or changes in histopathology are glucokinase regulatory protein, ornithine aminotransferase and cytochrome P450 (Cyp2C29) (14).
Various spectroscopic techniques have been applied in the past to biological fluids, with varying success (15-17). Raman spectroscopy has several attractive features that render it wellsuited for this type of analysis, including the relatively specific observed spectral bands as compared to UV/VIS absorption and fluorescence spectroscopy. In contrast to IR spectroscopy, Raman spectroscopy has the advantage of little interference from the $\mathrm{OH}$ stretches of water, making it more suitable for use in aqueous solutions.

We found that the data contained in the Raman spectra of the perfusates were well correlated with oxidative stress. We were able to reduce the data to approximately 3-4 independent dimensions, suggesting that we were monitoring 3-4 independent effects. The coefficients of the individual principle components tended to be monotonic with time. Further studies identifying the specific chemical entities that cause the spectral changes would be beneficial, as those specific spectral signatures could be used to guide the dimensionality reduction procedure. While PCA is effective at producing an orthogonal, highly reduced space, individual components are often difficult to interpret. More data are needed to validate the signatures we found; however, the difficulty in obtaining human livers makes a large dataset infeasible.

This preliminary work demonstrates that it is possible to monitor normothermic perfusion of human livers in real-time using Raman spectroscopy, potentially assessing the viability of the organ without offline measurements. Changes in the spectral signatures with time due to changes in the perfusate composition were well correlated with organ health and ORP, and could prove to be a valuable tool for assessing real-time drug toxicity, as well as organ viability prior to transplant. This approach could also be used to optimize perfusion conditions to lengthen harvest to transplant time, while still maintaining graft function.

\section{In memorium}

The authors would like to dedicate this manuscript to the memory of our beloved colleague, C. Gerald Curtis. Your scientific intellect and friendship will be greatly missed.

\section{References}

1. Krell H, Metz J, Jaeschke H, Hoke H and Pfaff E: Drug-induced intrahepatic cholestasis: characterization of different pathomechanisms. Arch Toxicol 60: 124-130, 1987.

2. Dahm LJ, Schultze AE and Roth RA: An antibody to neutrophils attenuates alpha-naphthylisothiocyanate-induced liver injury. J Pharmacol Exp Ther 256: 412-420, 1991.

3. Hill DA, Jean PA and Roth RA: Bile duct epithelial cells exposed to alpha-naphthylisothiocyanate produce a factor that causes neutrophil-dependent hepatocellular injury in vitro. Toxicol Sci 47: 118-125, 1999.

4. Rael LT, Bar-Or R, Aumann RM, Slone DS, Mains CW and Bar-Or D: Oxidation-reduction potential and paraoxonasearylesterase activity in trauma patients. Biochem Biophys Res Commun 361: 561-565, 2007.

5. Savitzky A and Golay MJE: Smoothing and differentiation of data by simplified least squares procedures. Anal Chem 36: 1627-1639, 1964

6. Hubert $\mathrm{M}$ and Engelen S: Robust PCA and classification in biosciences. Bioinformatics 20: 1728-1736, 2004.

7. Mwense M, Wang XZ, Buontempo FV, Horan N, Young A and Osborn D: QSAR approach for mixture toxicity prediction using independent latent descriptors and fuzzy membership functions. SAR QSAR Environ Res 17: 53-73, 2006. 
8. Stadler M, Nuyens V, Seidel L, Albert A and Boogaerts JG: Effect of nutritional status on oxidative stress in an ex vivo perfused rat liver. Anesthesiology 103: 978-986, 2005.

9. Zhang W, Wang M, Xie HY, et al: Role of reactive oxygen species in mediating hepatic ischemia-reperfusion injury and its therapeutic applications in liver transplantation. Transplant Proc 39: 1332-1337, 2007.

10. Lauschke H, Olschewski P, Tolba R, Schulz S and Minor T: Oxygenated machine perfusion mitigates surface antigen expression and improves preservation of predamaged donor livers. Cryobiology 46: 53-60, 2003.

11. Minor T, Manekeller S, Sioutis M and Dombrowski F: Endoplasmic and vascular surface activation during organ preservation: refining upon the benefits of machine perfusion. Am J Transplant 6: 1355-1366, 2006

12. Bessems M, 't Hart NA, Tolba R, et al: The isolated perfused rat liver: standardization of a time-honoured model. Lab Anim 40: 236-246, 2006
13. Iwai $\mathrm{M}$ and Shimazu T: Exaggeration of acute liver damage by hepatic sympathetic nerves and circulating catecholamines in perfused liver of rats treated with D-galactosamine. Hepatology 23: 524-529, 1996

14. Spicker JS, Pedersen HT, Nielsen HB and Brunak S: Analysis of cell death inducing compounds. Arch Toxicol 81: 803-811, 2007.

15. Enejder AM, Scecina TG, Oh J, et al: Raman spectroscopy for noninvasive glucose measurements. J Biomed Opt 10: 031114 2005.

16. Rohleder D, Kiefer W and Petrich W: Quantitative analysis of serum and serum ultrafiltrate by means of Raman spectroscopy. Analyst 129: 906-911, 2004

17. Rohleder D, Kocherscheidt G, Gerber K, et al: Comparison of mid-infrared and Raman spectroscopy in the quantitative analysis of serum. J Biomed Opt 10: 031108, 2005. 